\title{
Erratum to: Thioredoxin-Interacting Protein: a Novel Target for Neuroprotection in Experimental Thromboembolic Stroke in Mice
}

Tauheed Ishrat • Islam N. Mohamed • Bindu Pillai •

Sahar Soliman - Abdelrahman Y. Fouda • Adviye Ergul •

Azza B. El-Remessy • Susan C. Fagan

Published online: 11 December 2014

(C) Springer Science+Business Media New York 2014

\section{Erratum to: Mol Neurobiol}

DOI 10.1007/s12035-014-8766-x

The original paper of this article unfortunately contain errors on Figure 4. The images at sham and eMCAO/
TKO group panels are the same for both DAPI staining and caspase- 1 staining of the sham versus the eMCAO/ TKO.

The authors are sorry for these errors and certainly would like to correct the figure. Figure 4 is hereby corrected.

The online version of the original article can be found at http://dx.doi.org/ 10.1007/s12035-014-8766-X.

T. Ishrat $\cdot$ I. N. Mohamed $\cdot$ B. Pillai $\cdot$ S. Soliman $\cdot$ A. Y. Fouda $\cdot$

A. Ergul $\cdot$ A. B. El-Remessy $\cdot$ S. C. Fagan

Charlie Norwood VA Medical Center, Augusta, GA, USA

T. Ishrat $(\varangle) \cdot$ I. N. Mohamed $\cdot$ B. Pillai $\cdot$ S. Soliman $\cdot$

A. Y. Fouda · A. Ergul $\cdot$ A. B. El-Remessy $\cdot$ S. C. Fagan

Program in Clinical and Experimental Therapeutics, University of

Georgia College of Pharmacy, HM 1212; 1120 15th St., Athens,

GA 30912, USA

e-mail: tauheedarshi@gmail.com

T. Ishrat

e-mail: tishrat@gru.edu

A. Ergul

Department of Physiology, Georgia Regents University, Augusta,

GA, USA

S. C. Fagan

Department of Neurology, Georgia Regents University, Augusta,

GA, USA

A. B. El-Remessy

Culver Vision Discovery Institute, Georgia Regents University,

Augusta, GA, USA 
Fig. 4 TXNIP inhibition attenuates NLRP3 inflammasome protein activation at $24 \mathrm{~h}$ posteMCAO. Representative and quantitative analysis of Western blots (a) and

immunohistochemical representation around the penumbra (b) showing that NLRP3 expression and immunopositive signals increased after eMCAO and mitigated in RES-treated WT and TKO mice. Representative and quantitative analysis of Western blots of cleaved caspase-1 (c) and cleaved IL-1 $\beta$ (e) and increased and immunohistochemical representation of caspase-1 (d) after eMCAO. The expression of these proteins was attenuated in REStreated WT and TKO mice compared to vehicle-treated WT. Values are expressed as mean \pm SEM $(n=4-6, * P<0.05$

WT-shams vs WT-eMCAO,

$* P<0.05$ WT-eMCAO vs.

RES-WTeMCAO or

TKO-eMCAO)

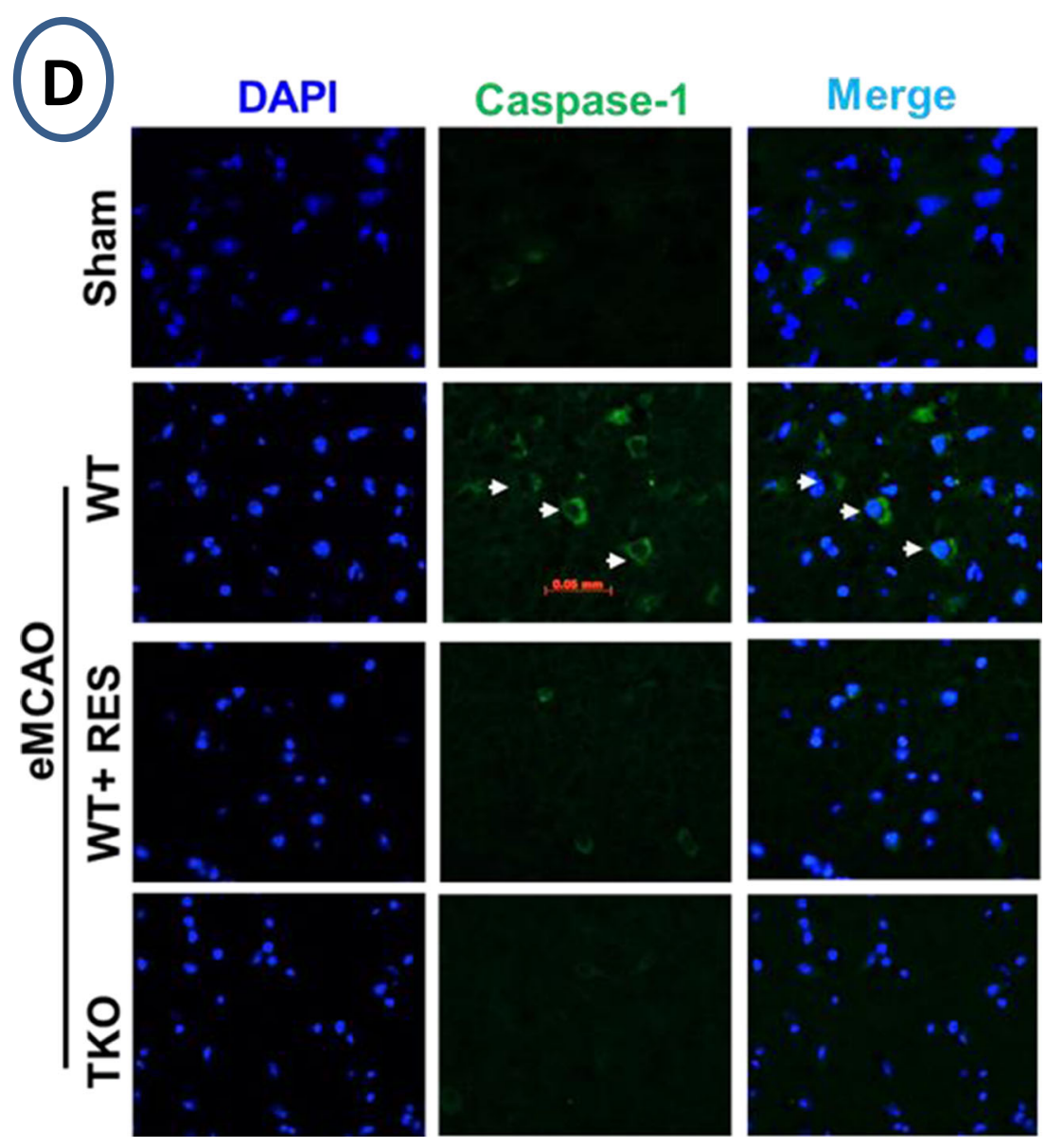

